\title{
Stochastic modelling of transition dynamic of mixed mood episodes in bipolar disorder
}

\author{
Yashaswini Kunjali Ajeeth Kumar, Aditya Kishore Saxena \\ Department of Computer Science and Engineering, School of Engineering, Presidency University, Bangalore, India
}

\begin{abstract}
In the present state of health and wellness, mental illness is always deemed less importance compared to other forms of physical illness. In reality, mental illness causes serious multi-dimensional adverse effect to the subject with respect to personal life, social life, as well as financial stability. In the area of mental illness, bipolar disorder is one of the most prominent type which can be triggered by any external stimulation to the subject suffering from this illness. There diagnosis as well as treatment process of bipolar disorder is very much different from other form of illness where the first step of impediment is the correct diagnosis itself. According to the standard body, there are classification of discrete forms of bipolar disorder viz. type-I, type-II, and cyclothymic. Which is characterized by specific mood associated with depression and mania. However, there is no study associated with mixed-mood episode detection which is characterized by combination of various symptoms of bipolar disorder in random, unpredictable, and uncertain manner. Hence, the model contributes to obtain granular information with dynamics of mood transition. The simulated outcome of the proposed system in MATLAB shows that resulting model is capable enough for detection of mixed mood episode precisely.
\end{abstract}

This is an open access article under the CC BY-SA license.

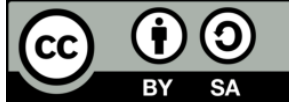

\section{Corresponding Author:}

Yashaswini Kunjali Ajeeth Kumar

Department of Computer Science and Engineering, School of Engineering, Presidency University

Bangalore, India

Email: yashaswini@presidencyuniversity.in

\section{INTRODUCTION}

Bipolar disorder can be stated as a type of psychiatric illness which is accompanied by various episodes right from depressive to hypomania [1]. Conventionally, this disorder is of various types viz. i) type-I bipolar disorder which is characterized by extreme mood of either manic or depression, ii) type-II bipolar disorder which is characterized by either hypomanic or depressive mood, and iii) cyclothymic disorder which is usually characterized by symptoms of depression and hypomanic but they do not exhibit depressive moods [2]. In order to standardize the process of identification, the standard authority called as International Classification of Diseases $10^{\text {th }}$ Edition i.e. ICD-10 is used for classification purpose [3]. According to this standard body, there is no significance difference between type-I and type-II bipolar disorder. The best representation can be given by ICD-10 when there are two significant episodes of mood where manic episodes are mandatory to be included in order to diagnose bipolar disorder [4]. There are different forms of clinical biomarkers which are used in diagnosis process where neuroimaging contributes to understand the distinction between unipolar and bipolar depression [5]. However, it is strongly felt that this distinction is never clear enough for understanding the complexities of mood transition in bipolar disorder. There are various existing approaches [6]-[10], where investigations are carried out towards bipolar disorder in order to improve the diagnosis process in the form of detection and classification mainly. At present, 
approaches related to imaging and pattern recognition are deployed in order to extract the essential information of the neurons along with their biological operation. This information assists in ascertaining the current condition of subject on the basis of behavioral scale. However, unique fact to observe here is that there are no significant studies being carried out considering a subject with mixed moods in bipolar disorder. In all the above-mentioned standard taxonomies of bipolar disorder, there is a clear characteristic of every specific type of this disorder; however, the challenging part in mixed mood is that they are accompanied by combination of all possible moods of bipolar disorder. This exhibit of combined and different mood transition makes the diagnosis process very much challenging [11]. One of the challenging aspects of mixed mood episodes is to find out the pattern of transition of rate of change of mood with respect to time. Absence of this state of transition pattern makes the diagnosis modelling quite challenging. Therefore, the proposed system addresses this problem by developing a unique modelling of mixed mood episodes using stochastic approach. The proposed system is also capable of reproducing different stages of stimulation in order to differentiate different form of episodes in behavioral stimulation. The main contribution of the proposed study are viz: i) it presents a simplified mathematical modelling of behavioral stimulation and its association with mood transition, ii) it is completely an involuntary process of control and response system towards different forms of mood obtained by continuous stochastic data distribution process, iii) it connects nonlinearity with the dynamics of mood transition to offer higher stabilized outcome, and iv) the proposed mathematical model is clearly developed for offering granular information about mixed mood in bipolar disorder. The organization of this manuscript is as follows: section 2 discusses about existing approaches towards bipolar disorder, section 3 discusses about the research problems derived from prior section. Briefing of proposed method is carried out in section 4 while section 5 illustrates about the proposed analytical model. Section 6 discusses about simulation outcome while section 7 summarizes the paper.

\section{RELATED WORK}

Majority of the existing investigation towards bipolar disorder is associated with detection of its level in order to assist in diagnosis process. Most recently, bipolar disorder is classified into two significant forms i.e. depressive and unipolar disorder and investigation is carried out to identify it Huang et al. [12]. This study analyzes the speech response of the subject where spectral clustering is further applied to achieve non-biased classification. Adoption of spectral clustering is also alongside with temporal information in order to detect mood disorder. Identification of specific mood was also reported to be carried out using features extracted from audio and video in $\mathrm{Su}$ et al. [13]. Implementation of the manifold learning scheme is also witnessed to offer significant detection of mental disorders Liu et al. [14]. When learning is carried out over multiple instances, the chances of effective diagnosis are further ascertained Ren et al. [15]. However, these studies introduce a model which requires the system to be configured and fine-tuned on the basis of fed input. Hence, the next revolution of the study is towards involuntary detection process toward various neurologic diseases Nunes et al. [16]. The study also introduces a decision-making concept over multicriteria in order to assess the mental disorder. Involuntary scheme is further investigated along with inclusion of different signal modalities Syed et al. [17]. Adoption of encoding with Fisher vector to obtain feature is proven to extract feature from screen data of bipolar disorder. Existing researchers finds that there are pitfalls associated with investigating mechanism of the features using unsupervised approach. This issue can be addressed by using generative model of deep learning over radiological data Matsubara et al. [18]. The study focuses on identification of bipolar disorder and schizophrenia. Adoption of various significant features considering the steady state of the visual data can further contribute towards this classification process Alimardani et al. [19]. The study performs classification on the basis of brain activity data subjected to supervised learning scheme.

It is seen that much emphasis is carried out towards identification of depression state in bipolar disorder. Existing study witnesses the usage of geographic location data for this purpose Palmius et al. [20]. A discriminant classification over linear model of regression is designed to carry out this form of detection. Apart from detection-based approach, there is also recognition-based approach. Temporal factor is considered as prominent indicator towards this process. Existing approach reports of using temporal representation of signal in discriminative manner for recognizing the state of bipolar disorder in Du et al. [21]. Yang et al. [22] an interesting study has been carried out towards using gestures of subject for identifying bipolar disorder. The study extracts histogram from the body posture of a subject along with usage of recurrent neural network and random forest in order to carry out classification. At present, the trend of the study towards investigating bipolar disorder is essentially predictive scheme viz. signature-based model in Kormilitzin et al. [23], using nonlinear dynamics in Valenza et al. [24], Trotzek et al. [25] usage of linguistic approach using neural network, and Alghamdi et al. [26] applying natural language processing over social network. Majority of the predictive scheme for bipolar disorder is carried out considering machine learning dominantly. With a purpose of achieving higher accuracy in detection and classification process, some of the significant machine-learning based approaches are viz. identifying depression from audio using convolution neural network in Wang et al. 
[27], Jazaery and Guo [28] applying deep learning on spatio-temporal attributes, Ding et al. [29], and Tadesse et al. [30] support vector machine over textual data, Cao et al. [31] deep neural network for investigate the possibility of bipolar disorder on mobile usage, integrated usage of K-nearest neighborhood, support vector machine, Mahendran et al. [32] random forest and multi-layer perceptron for constructing generalized model for stacking. The above-mentioned studies offer a productive guideline for modeling bipolar disorder while the pitfalls associated with it is highlighted in next section.

\section{PROBLEM STATEMENT}

Existing studies has a straight forward approach of identifying the essential characteristics of bipolar disorder with presumption of the subject data. The pre-defined data of a subject is further subjected to various existing approaches as discussed in prior section in order to identify the depression. However, there are very few work that has been carried out towards mixed mode of states in bipolar disorder. Studies are more focused on type-I and type-II bipolar disorder but not on mixed features. In order to extract precise information about the mental state of subject, it is necessary to investigate the moods and their dynamic state of transition. Unfortunately, there are no standard model or benchmark studies being carried out in this direction.

In order to understand the problem associated with the dynamics of mood in mixed mode of bipolar disorder, consider an example: Assume that $v_{1}, v_{2}, v_{3}$, and $v_{4}$ are four discrete states of mood (say high energy, insomnia, restlessness, irritable). Mathematically, it will also mean that $v_{1} \neq v_{2} \neq v_{3} \neq v_{4}$. An empirical representation of dynamic transition of mood of a subject with mixed feature will be $v_{1} \rightarrow \operatorname{random}\left(v_{2}, v_{3}, v_{4}\right)$. A proper treatment in this situation can be carried out if this transition is known. However, this formulation of dynamic transition of mood is a direct exhibit of a distribution of random probability which are not possible to perform accurate prediction. It will eventually mean that existing approaches adopting predictive approaches will definitely not be applicable in mixed features of bipolar disorder. However, a stochastic mathematical modelling can solve this if integrated with probability and statistics, which has not been explored yet.

Another significant finding from problem formulation is that the variables $v_{1}, v_{2}, v_{3}$, and $v_{4}$ is a direct representation of behavioral stimulation which has not been reported to be used in any existing modelling. Although, existing approaches deals with detection of depression states majorly in bipolar disorder, but very often manic and euthymia is not studied at same time as the focus is towards type-I or type-II detection. Existing studies have also never reported to emphasize on extrinsic and intrinsic factors towards transition of states of mood. Apart from this directly connecting mood with behavioral stimulation could also lead to outliers. Extrinsic factors e.g. healing time of subject would have an impact on next round of attacks either episodes of bipolar or manic. Intrinsic factor e.g. dynamic changes in the mood is a direct representation of nonlinearity in the system has not be part of modelling yet, which could be one prime indicator of dynamics of mood in bipolar disorder.

From the modelling viewpoint, existing approaches are found to include complex form of modelling with no update over the parameters. This is a direct indication that existing models doesn't support dynamicity associated with the mood transition associated with bipolar disorder. This problem is solved in proposed system using a simplified mathematical model briefed in next section.

\section{PROPOSED SOLUTION}

The prime idea of the proposed system is to develop a simplified analytical model of transition of temperament patterns of a subject suffering from bipolar disorder. The proposed system makes use of behavioral stimulation of a subject and formulates it using series of sequential operation as shown in Figure 1. The core agenda of the proposed architecture is to formulate the fact that maximized cases of nonlinearities over the system will eventually generate enhanced steadiness of system. It also investigates various levels of transition of the mixed-level mood of a subject with bipolar disorder. The system model formulates a data mapping with behavioral stimulation in order to generate various latent attributes associated with dynamics of this disorder. The outcome of the study contributes to find the reason associated with time and rate of healing period of patient with bipolar disorder. The proposed study implements the concept using mathematical model.

The proposed study depicts the behavioral stimulation in the form of involuntary stimulation mechanism that controls various behavioral stimuli in order to showcase various characteristics of nonlinearity. The model also contributes to the fact that alteration in the system linearity is adequate to formulate various transition states among dynamics of behavioral stimulation in bipolar disorder. The architecture initiates with developing stimulation response system as well as controlling mechanism of involuntary type. The stimulating response system is further classified into confident and adverse response system with various dependable attributes. Rate computation is carried out to check the speed of transition of mood patterns associating with behavioral stimulation. In order to make the system information more granular, decomposition towards the rate 
is studied followed by updating operation at the end. The complete sequence of operation leads to successful modelling of behavioral stimulation of a subject with bipolar disorder. Illustration of the architecture and the respective operation is carried out in next section.

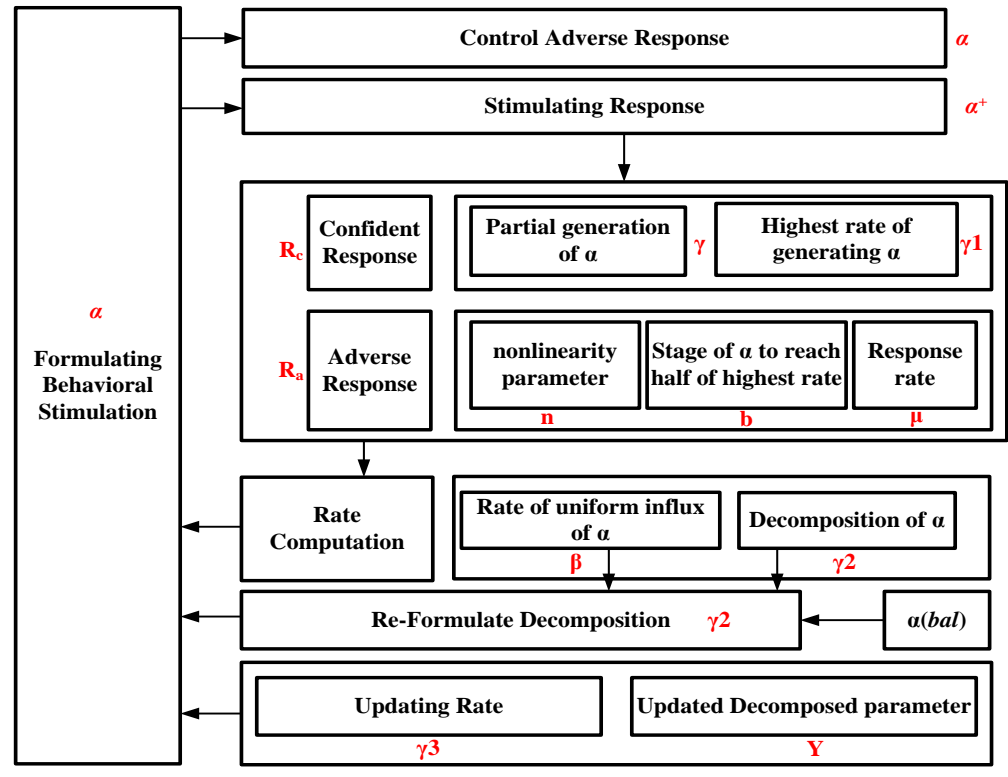

Figure 1. Proposed architecture for behavioral stimulation

\section{PROPOSED METHOD}

The proposed system performs modelling of comprehensive bipolar disorder considering the stimulating stages of various behavior of the subject. The study considers a parameter $\alpha$ that represents stages of stimulating behavior and it represents the degree of interactivity that the subject has with the environment. The proposed model is not about obtaining inference about the intensity of the mood from the stages of behavioral stimulation but to carry out a comprehensive modelling of various significant stimulation of behavior. As an outcome, such stimulating stages are exhibited to be connected with 3 essential episodes of depression i.e. i) depressive, ii) hypomanic, and iii) manic. The study is more inclined towards investigating mixed form of episodes that occurs during bipolar disorder. The study also chooses to obtain its findings that the occurrence of either hypomanic episodes or manic could result in secondary behavior associated with aggression. This could be acting as a significant outcome of proposed behavioral stimulation approach.

The proposed modelling considers two forms of response system which is responsible for regulating $\alpha$ i.e. stages of behavioral stimulation viz. i) involuntary stimulating response and ii) involuntary adverse response. The first form of response system is about using the stages of behavioral stimulation and using them for involuntary stimulation. This response is signified as $\alpha^{+}$. The second form of the response system is about to retain the parameter $\alpha$ at the stages of normal activity. Therefore, the parameter $\alpha$ is considered as an element which is generated as well as decomposed while working on particular process. It should be noted that the proposed system targets to investigate the dynamic connectivity between the target parameters in order to investigate the behavioral stimulation of a subject. The secondary purpose of this approach is to appropriately control the computational complexity associated with cumulative operation of the system in order to ensure the better applicability towards practical world scenario. Therefore, the proposed model considers that $\beta$ is a rate of uniform influx for seamless occurrence of behavioral stimulation $\alpha$ while $\gamma_{2}$ is considered as decomposition rate of behavioral stimulation $\alpha$. The uniform rate of influx $\beta$ basically estimates the level of baseline over a constant point. It will mean that if the value of $\beta$ is equivalent to 0 than constant point at lower level will be equivalent to score of $\alpha$ equivalent to 0 too. This scenario will be equivalent to stimulation stage that doesn't exist. However, this fact cannot alter the behavior of the system in qualitative manner and therefore the proposed system opts for $\beta$ to be a rate to guarantee the reduced fluctuation in the stage for $\alpha$ in positive manner. Mathematically, the changes in behavioral stimulation can be represented as:

$$
\frac{d(\alpha)}{d t}=\delta_{1}-\delta_{2}
$$


The expression (1) is used for representing alteration in behavior of the system using stages of behavioral stimulation $\alpha$. The variable used are $\delta_{1}$ and $\delta_{2}$ which corresponds to (2).

$$
\begin{aligned}
& \delta_{1} \rightarrow R_{c}+R_{a}+\beta \\
& \delta_{2} \rightarrow \gamma_{2} \cdot \alpha
\end{aligned}
$$

In the expression (2), the variables $\mathrm{R}_{\mathrm{c}}$ and $\mathrm{R}_{\mathrm{a}}$ represents confident response and adverse response considering stage of behavioral stimulation $\alpha$.

The mathematical expression associated with the involuntary stimulation $\alpha$ with respect to confident response $R_{c}$ is now represented as (3):

$$
R_{c}=H_{1} \cdot H_{2}
$$

In the expression (3), the variable $\mathrm{H}_{1}$ and $\mathrm{H}_{2}$ represents $\left(\gamma^{l}+\alpha^{l}\right)^{-1}$ and $\gamma_{1}$. $\alpha^{l}$. The variable $\gamma$ represents a partial rate which means estimation of the rate where the stage of the behavioral stimulation is considered as half of the highest possible rate. The variable $\gamma_{1}$ represents highest rate associated with a yield of involuntary stimulation $\alpha$ while the variable $l$ represents a parameter associated with nonlinearity. The proposed study considers the variable $l$ which is responsible for controlling the curve of stimulation i.e. $\mathrm{R}_{\mathrm{c}}$. It will mean that if there is a reduced score of non-linearity than the system will eventually generate a dynamic of hyperbolic response associated with $\mathrm{R}_{c}$, while response of sigmoidal shape is generated for maximized stages of nonlinearity. Therefore, it can be seen that degree of responsiveness is found to vary as the levels of stages of behavioral stimulation $\alpha$ for lower value and $\gamma$ for higher value of response. It will also mean that there is specific part of mood control system where sensitivity differs from each other. Therefore, this fact is in agreement with the hypothesis that behavioral stimulation system cannot be effectively controlled by the subjects suffering from the bipolar disorder. Hence, it is not a wise decision to associate depression with passive behavioral stimulation system or manic in case of higher activity of behavioral stimulation for a subject. The proposed system also emphasizes over the adverse response system in order to retain the equilibrium condition associated with conventional stages of stimulation. The mathematical expression of the adverse response system $\left(R_{a}\right)$ is represented as (4):

$$
R_{a}=\mu(c-\theta)
$$

In the expression (4), the variable $\mu$ represents the response rate while the coefficient $c$ is considered for signifying the strength of the response rate. Therefore, where the value of $c=1 / 2$, then the overall variable of $\mu$.c will represent highest possible response rate. The second component of the expression (4) $\theta$ represents $\left(1+\mathrm{e}^{\mathrm{n} . \mathrm{d}}\right)^{-1}$ where $n$ is parameter for non-linearity and the parameter $d$ is equivalent to $(b-\alpha)$. The variable $\mathrm{b}$ represents the overall value of $\alpha$ which is equivalent to partially accomplished rate. The proposed study considers $\alpha$ as the normal stimulus stage. The operation carried out by non-linear coefficient $n$ performs the similar operation in the adverse response as seen by the parameter of confident response $R_{c}$ with respect to the shape of the function of response. This is done is such a way that sigmoidal functions of response are found for increasing value of $n$ while linear function of response is found for lower values of $n$. The proposed implementation performs selection of equivalent values of $b$ and $\gamma$. The study also performs construction of a decomposition parameters $\gamma_{2}$ which is defined as a score to achieve the balanced state of confident response at $\alpha(\mathrm{bal})$ considering the value of $l$ as unity. The mathematical expression for this new decomposition parameter $\gamma_{2}$ is stated as (5):

$$
\gamma_{2}=v_{1}+v_{2}
$$

In the expression (5), the first component $v_{1}$ represents $\gamma_{1} /(\gamma+\alpha(b a l))$ while the second component $v_{2}$ represents $\mathrm{b} / v_{1}(\mathrm{bal})$. The expression (5) will mean that position of the non-steady point will reside over $v_{1}(\mathrm{bal})$ at higher values of the parameter of non-linearity i.e. $l$. This parameter is maximized in order to permit the involuntary control of response system. The proposed system achieves steadiness of this non-steady parameter by using adverse response system. Therefore, this operation will lead to various degree of stabilization targets with higher, lower, and medium stages of stimulation. It is tentative that the adverse response could be possibly considered to be equivalent to various attempts of behavior of a subject in order to resists the higher stages of behavioral stimulation. This phenomenon also states that this system will be less effective as the response system saturates towards its higher and lower stages. It is definitely not adequate in order to balance the stages of stimulation which are higher than the cut-off value controlled by the dynamic characteristic of system. The prime reason for susceptibility of the behavioral stimulation system could be caused owing to the incorporation 
of a parameter $\mathrm{Y}$ that is decomposed by the stages of $\alpha$ considering a rate of decomposition with $\gamma_{3}$. It is to be understood here that all the challenging events are not required to be linked with adverse direction towards $\mathrm{Y}$ parameter. This hypothesis also leads to a conclusion that challenging events can also contribute towards maximization of stimulation in behavior $\alpha$. The assessment is carried out on both the positive as well as negative direction of $\mathrm{Y}$ in order to confirm that proposed system should emphasize on all the forms of parameters that could possibly influence stimulation in behavior $\alpha$. The proposed system further models that:

$$
\begin{aligned}
& \frac{d\left(\alpha_{1}\right)}{d t}=\frac{d(\alpha)}{d t}+\gamma_{3} . Y \\
& \frac{d(Y)}{d t}=-\gamma_{3} . Y
\end{aligned}
$$

In the above first expression (6), the initial component is a substituted value of expression (1). The proposed system, therefore, explores various mechanisms in order to state that there are multiple extrinsic and intrinsic factor that contributes towards the stimulation states of a subject in bipolar disorder. This is a unique finding which will offer more granularities in understanding the underlying states of a subject associating with an event of various environmental stimulation facts. The next section discusses about the study outcomes.

\section{RESULT AND DISCUSSION}

The proposed system makes use of Euler's mechanism for approximation of the numerical solution which is associated with equation of stochastic nature. For all the expression specified in prior section, the proposed system adds noise and used a step-size for time as its square root in adherence with normal distribution. The proposed study is scripted in MATLAB where probability density is chosen to represent normal distribution (i.e. normal mood) which is a spontaneous process of all the behavioral stimulation. The continuous value of the distribution is extracted by the amplitude associated with the event distribution. In order to carry out observation, the proposed system introduces a parameter $\rho$ which acts like an influencing parameter associated with dynamics of stimulation. The assessment is carried out over time-series data. The study also makes use of case study where the day-wise observation is carried out by displacing a mean window of one week. All the numerical data bounds are recorded for the mean stimulation stages associated with this window. Therefore, the state transition of the data $\rho$ is mathematically represented as (7):

$$
\rho=\psi^{2} \cdot \pi
$$

In the above expression, the variable $\psi$ represents quantified mean windows which is basically equivalent to the cumulative simulation time for 24 hours (duration) subtracted by the window size (i.e. 7). The second variable $\pi$ represents inverse of summation of squared value of $l_{\mathrm{s}}$ i.e. the number of events identified out of multiple level of stimulation. For an example: if the mean of the window is within 80, it will represent depression or minimal stimulation. If the mean of the window is more than 120, it will represent mania or higher stimulation while values between 80-120 will represents medium stimulation. The study considers a scale of stimulation stage where a random unit of such values is considered. By adopting this analytical process, the proposed system offers greater deal of precision in understanding and separating the values with increasing fluctuation that are extracted from the noise proportion. For the purpose of assessment of proposed system, the simulation in MATLAB is carried out for unit stochastic process over one subject on time-series data. The analysis is also carried out over dynamic attributes, proportion of noise, and quantity of changing events over each simulation. Mean of all the data are obtained in this process of analysis. The proposed system fixed the noise value as 0.1 and captures all the probability information associated with an event. The study also fixes the maximized amplitude of the event of random distribution to be 0.01 . In order to offer a clear inference of the proposed simulation out, the analysis is carried out with a hypothesis that extent of the non-linearity associated with involuntary stimulation system is basically equivalent to different stages of bipolar disorder. It will eventually mean that if the value of the $l$ parameter is equivalent to unity than it will represent subject with less significant bipolar disorder. However, if the value for same parameter is found more than unity than it represents subject with critical stage of bipolar disorder. At the same time, if the subject is identified to have positive bipolar disorder than increasing value of same parameter $l$ will corresponds to historical records of manic or depressive events. However, the model doesn't offer any form of predictive operation for highlighting the mechanism of progressive trend in distribution curve with nonlinearity. The proposed model is only responsible for extracting the precise information associated with the behavior of the system at a defined value of $l$ parameter. An extensive analysis is carried out for proposed model over different value of $l$ parameter in order to show distinctly three possible cases of bipolar disorder i.e. i) depression, ii) mania, and iii) Euthymia. The stability of the system increases with the maximized value of $l$ parameter. The impacts of different 
parameters over different test parameter are showcased in Figure 2 to Figure 5. The prime dependable parameter that is significantly influenced by stages of $l$ parameter is $\gamma$ and $\delta$ mainly. The scope of the stability for the score in an intermediate stage of stimulation is evaluated from non-linearity coefficient $n$ associated with the adverse response signifying the different sets of corresponding behavior during changes of mood. Maximized value of non-linearity coefficient $n$ increases this scope to maximized value of $l$ while it also results in minimization of numerical scope between the intermediate and higher stability ranges. While initiating the assessment, it is anticipated that proposed model should be highly robust in case of any events of the alterations in the system parameters. One example to cite this fact is that possibility of the enhanced outcome of stability on increased vale of $l$ will take place when system reduces the $\gamma_{1}$ parameter. Therefore, the system might demand increased value of $l$ in order to pay off this effect for retaining similar performance outcome. On the other hand, when an involuntary control of the behavioral stimulation is carried out with an aid of adverse response than the system is found to be efficient for almost any value of the $l$ parameter. The indicator of this control system of the distribution curve is basically similar to confident response of the system. This tendency of the curve represents a mechanism to resist any form of inappropriate variance of stages of stimulation associated with the different stages of subject's mood. Hence more granularities in the data as well as its inference is obtained for bipolar disorder.

Figures 2 and 3 highlights the highlights the graphical analysis for normalized mood captured in increasing week time and probability density over different values of normalized values of mood respectively. According to this outcome, it can be stated that the increase in episodes of bipolar disorder recorded in history will eventually demand increasing time to heal up from the adverse symptoms of bipolar disorder. This fact can be checked by maximizing the value of $l$ parameter of nonlinearity. The outcome also shows that an amendment in system non-linearity will also lead to extraction of various granular information about the behavioral changes under different stages of stimulation. The assessment is carried out for time-series data in order to evaluate the rate constants for the decomposition of the stimulation stages used in the analysis. This can be represented using the parameter $\gamma_{3}$ which basically depicts the decomposition of the stimulation stages over domain of time after the event has already occurred as shown in Figures 2 and 3. The output curve highlights the normalized value associated with the number of subjects that are found to be more than the median value of behavioral stimulation stages. An exponential decomposition is found in the temporal trajectory of the utilized data that offers a rationale of the adoption of parameter $\gamma_{3}$, which reflects the occurrence of various events.

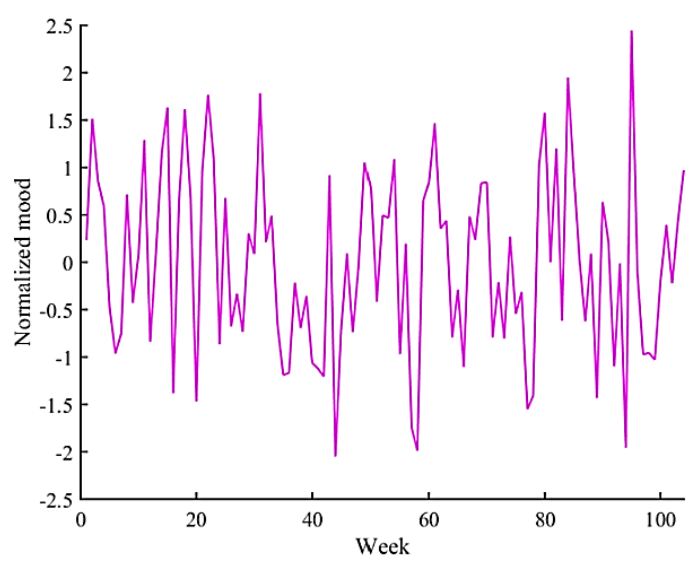

Figure 2 Analysis of normalized mood per week

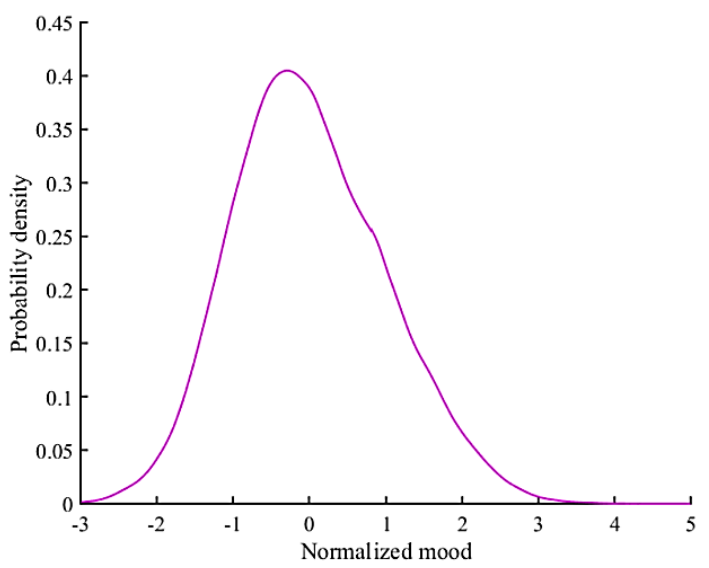

Figure 3 Analysis of probability density in varying normalized mood

The study outcome in Figure 2 highlights that the proposed system is capable of effectively capturing the information associated with the normalized mood. A closer look into the proposed system highlights that proposed system is capable of exploring the intermediate states of bipolar disorder where apart from higher state with lower stimulation (Depression) and higher state with higher stimulation (Mania) is identified along with the Euthymia state which is represented by intermediate state of stimulation as shown in Figure 4 . While Figure 3 highlights that probability distribution of normalized mood to exhibit Gaussian distribution pattern exhibiting the reliability of the proposed system. The study has considered the 10 units for any form of disturbances that may be possibly present within behavioral stimulation in the presented simulation process. It was found that increasing probability density curve to go back to the descent of normalized mood axis after the 
system model encounters any form of significant events of stimulation associated with the higher or lower states of stimulation. The next part of the assessment is about survival probability and spectral density with respect to duration of 24 hours. Basically, the term survival probability corresponds to the duration of the state of stimulation associated with 3 mixed mode states i.e. depression, mania, and euthymia. A closer look at both the outcomes in Figures 4 and 5 shows that proposed system is capable of generating significantly realistic course of time associated with multiple dynamics of moods associated with bipolar disorder. Spectral density assists in offering more significance of detection in presence of noise. The analysis shows that system encounter stabilized stimulation for reduced value of $l$ parameter irrespective of any form of alterations over the noises due to event or any other sensitive parameters i.e. $\mathrm{R}_{\mathrm{c}}$ or $\mathrm{R}_{\mathrm{a}}$. The outcome from Figure 5 also exhibits the possibility of any specific events that can translate its cause in the form of behavioral stimulation for any stages of $l$ parameter. This eventually states that the proposed system is capable of constructing a patter for mood swings during bipolar disorder with non-fluctuating ranges. They are also capable of exhibiting the normal transition from lower to higher to intermediate state of mood of a subject with cyclic pattern in its distribution curve. The study outcome also shows the accountability of different types of artifacts / noise towards fluctuating values of stimulating frequencies of different stochastic events. Hence, the proposed system establishes different behavioral stimulation points with the bipolar disorder

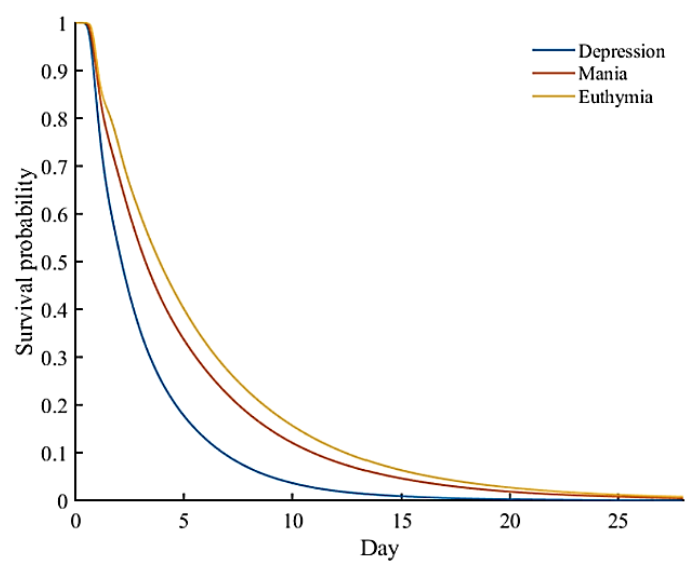

Figure 4. Analysis of survival probability per day

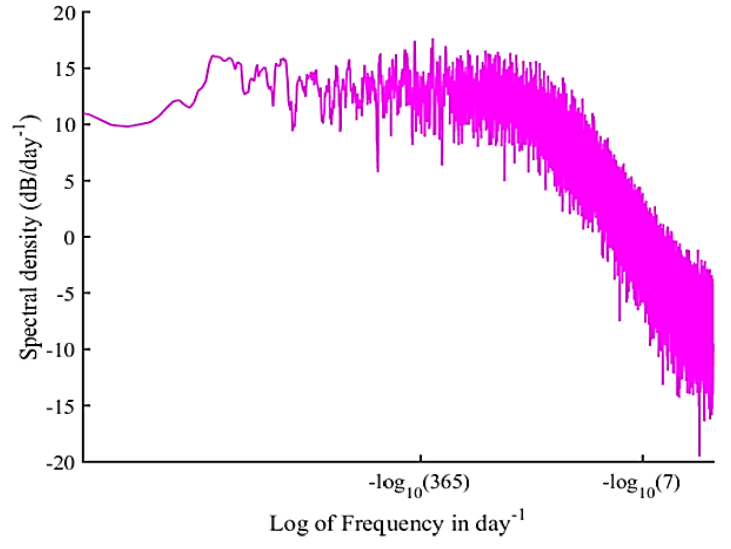

Figure 5. Analysis of spectral density per log of frequency per day

\section{CONCLUSION}

This paper presents a discussion about a simplified novel analytical model in order to offer a proper representation of dynamics involves in different behavioral stimulation control. This exploration of the spectrum of variational mood transition is meant for in-depth diagnosis of mixed mood episodes in bipolar disorder. The significant contribution of the proposed model is that it offers capability to showcase an exclusive distributive patterns of mood episodes stochastically associating with bipolar disorder. It consists of both steady as well as maximized transition of mood patterns under different scales of time. Unlike any existing system, the proposed system doesn't make use of any standard dataset associated with mood. Following are the justification behind this: i) adoption of predefined information about mood from existing dataset will only assists in validation of model but it could ensure the stability and consistency of the model when the database is changed and ii) existing dataset are mainly related to only one type of bipolar disorder and not much of mixed mode episodes. Therefore, the proposed system programmatically generates the dataset in the form of behavioral stimulation using random probability distribution which can be mapped with stochastic nature of transition of mood. Hence, the applicability of the proposed model is quite high for any practical world cases of bipolar disorder.

\section{REFERENCES}

[1] H. Belete, T. Ali, and G. Legas, "Relapse and clinical characteristics of patients with bipolar disorders in central ethiopia: A crosssectional study," Hindawi-Psychiatry Journal, vol. 2020, 2020, Art. no. 8986014, doi: 10.1155/2020/8986014.

[2] A. Ashraf, T. S. Gunawan, B. S. Riza, E. V. Haryanto, and Z. Janin, "On the review of image and video-based depression detection using machine learning," Indonesian Journal of Electrical Engineering and Computer Science (IJEECS), vol. 19, no. 3, pp. 1677-1684, 2020, doi: 10.11591/ijeecs.v19.i3.pp1677-1684.

[3] "ICD10Data," ICD10Data.com. https://www.icd10data.com/ICD10CM/Codes/F01-F99/F30-F39/F31-/F31.9 (accessed Jul. 15, 2021). 
[4] K. A. Yashaswini and S. Rao, "Bipolar disorder: A pathway towards research progress in identification and classification," In Computer Science On-line Conference, 2020, pp. 205-214, doi: 10.1007/978-3-030-51965-0_17.

[5] M. Z. Nasrudin, R. Maskat, and R. Musa, "Detecting candidates of depression, anxiety and stress through malay-written tweets: a preliminary study," Indonesian Journal of Electrical Engineering and Computer Science (IJEECS), vol. 16, no. 2, pp. 787-793, 2019, doi: 10.11591/ijeecs.v16.i2.pp787-793.

[6] S. Aich, M. I. Joo, H. C. Kim, and J. Park, "Improvisation of classification performance based on feature optimization for differentiation of Parkinson's disease from other neurological diseases using gait characteristics," International Journal of Electrical and Computer Engineering (IJECE), vol. 9, no. 6, pp. 5176-5184, 2019, doi: 10.11591/ijece.v9i6.pp5176-5184.

[7] N. Shetty, B. Muniyal, A. Anand, S. Kumar, and S. Prabhu, "Predicting depression using deep learning and ensemble algorithms on raw twitter data," International Journal of Electrical and Computer Engineering (IJECE), vol. 10, no. 4, pp. 3751-3756, 2020, doi: 10.11591/ijece.v10i4.pp3751-3756.

[8] H. Cai et al., “A pervasive approach to EEG-based depression detection,” Wiley Journals of Hindawi Complexity, 2018, doi: $10.1155 / 2018 / 5238028$

[9] B. Sulistyo, N. Surantha, and S. M. Isa, "Sleep apnea identification using HRV features of ECG signals," International Journal of Electrical and Computer Engineering (IJECE), vol. 8, no. 5, pp. 3940-3948, 2018, doi: 10.11591/ijece.v8i5.pp3940-3948.

[10] E. Z. Huerlimann, M. G. Holtforth, and E. Hermann, "Long-term effects of the treatment of depressive female inpatients in a naturalistic study: Is early improvement a valid predictor of outcome?," Hindawi Publishing Corporation Depression Research and Treatment, 2014, doi: 10.1155/2014/780237.

[11] N. S. Parameswaran and D. Venkataraman, "A computer vision based image processing system for depression detection among students for counseling," Indonesian Journal of Electrical Engineering and Computer Science (IJEECS), vol. 14, no. 1, pp. 503-512, 2019, doi: 10.11591/ijeecs.v14.i1.pp503-512.

[12] K. Y. Huang, C. H. Wu, M. H. Su, and Y. T. Kuo, "Detecting unipolar and bipolar depressive disorders from elicited speech responses using latent affective structure model," in IEEE Transactions on Affective Computing, vol. 11, no. 3, pp. 393-404, 2020, doi: 10.1109/TAFFC.2018.2803178.

[13] M. H. Su, C. H. Wu, K. Y. Huang, and T. H. Yang, "Cell-coupled long short-term memory with \$1\$ -skip fusion mechanism for mood disorder detection through elicited audiovisual features," in IEEE Transactions on Neural Networks and Learning Systems, vol. 31, no. 1, pp. 124-135, Jan. 2020, doi: 10.1109/TNNLS.2019.2899884

[14] W. Liu, D. Li, and H. Han, "Manifold learning analysis for allele-skewed DNA Modification SNPs for psychiatric disorders," in IEEE Access, vol. 8, pp. 33023-33038, 2020, doi: 10.1109/ACCESS.2020.2974292.

[15] Z. Ren, J. Han, N. Cummins, Q. Kong, and M. D. Plumbley, "Multi-instance learning for bipolar disorder diagnosis using weakly labelled speech data," Proceedings of the 9th International Conference on Digital Public Health, 2019, doi: $10.1145 / 3357729.3357743$.

[16] L. C. Nunes, P. R. Pinheiro, M. C. D. Pinheiro, M. S. Filho, R. E. C. Nunes, and P. G. C. D. Pinheiro, "Automatic detection and diagnosis of neurologic diseases," in IEEE Access, vol. 7, pp. 29924-29941, 2019, doi: 10.1109/ACCESS.2019.2899216

[17] Z. S. Syed, K. Sidorov, and D. Marshall, "Automated screening for bipolar disorder from audio/visual modalities," Proceedings of the 2018 on Audio/Visual Emotion Challenge and Workshop, Oct. 2018, doi: 10.1145/3266302.3266315.

[18] T. Matsubara, T. Tashiro, and K. Uehara, "Deep neural generative model of functional MRI images for psychiatric disorder diagnosis," in IEEE Transactions on Biomedical Engineering, vol. 66, no. 10, pp. 2768-2779, Oct. 2019, doi: 10.1109/TBME.2019.2895663

[19] F. Alimardani, J. Cho, R. Boostani, and H. Hwang, "Classification of bipolar disorder and schizophrenia using steady-state visual evoked potential based features," in IEEE Access, vol. 6, pp. 40379-40388, 2018, doi: 10.1109/ACCESS.2018.2854555.

[20] N. Palmius et al., "Detecting bipolar depression from geographic location data," in IEEE Transactions on Biomedical Engineering, vol. 64, no. 8, pp. 1761-1771, Aug. 2017, doi: 10.1109/TBME.2016.2611862.

[21] Z. Du, W. Li, D. Huang, and Y. Wang, "Bipolar disorder recognition via multi-scale discriminative audio temporal representation," Proceedings of the 2018 on Audio/Visual Emotion Challenge and Workshop, Oct. 2018, doi: $10.1145 / 3266302.3268997$.

[22] L. Yang, Y. Li, H. Chen, D. Jiang, M. C. Oveneke, and H. Sahli, "Bipolar disorder recognition with histogram features of arousal and body gestures," Proceedings of the 2018 on Audio/Visual Emotion Challenge and Workshop, Oct. 2018, doi: $10.1145 / 3266302.3266308$.

[23] A. Kormilitzin, K. E. A. Saunders, P. J. Harrison, J. R. Geddes, and T. Lyons, "Detecting early signs of depressive and manic episodes in patientswith bipolar disorder using the signature-based model," arXiv:1708.01206v1, Aug. 2017.

[24] G. Valenza, M. Nardelli, A. Lanata, C. Gentili, G. Bertschy, M. Kosel, and E. P. Scilingo, "Predicting mood changes in bipolar disorder through heartbeat nonlinear dynamics," IEEE Journal Of Biomedical And Health Informatics, vol. 20, no. 4, pp. 1034-1043, 2016

[25] M. Trotzek, S. Koitka, and C. M. Friedrich, "Utilizing neural networks and linguistic metadata for early detection of depression indications in text sequences," IEEE Transactions On Knowledge And Data Engineering, vol. 32, no. 3, pp. 588-601, 2020, doi: 10.1109/TKDE.2018.2885515

[26] N. S. Alghamdi, H. A. H. Mahmoud, A. Abraham, A. A. Alanzai, and L. Garcia-Hernandez, "Predicting depression symptoms in an arabic psychological forum," IEEE Access, vol. 8, pp. 57317-57334, 2020, doi: 10.1109/ACCESS.2020.2981834.

[27] Z. Wang, L. Chen, and G. Diao, "Recognition of audio depression based on convolutional neural network and generative antagonism network mode," IEEE-Access-Special Section On Emerging Deep Learning Theories And Methods For Biomedical Engineering, vol. 8, pp. 101181-101191, 2020, doi: 10.1109/ACCESS.2020.2998532.

[28] M. A. Jazaery and G. Guo, "Video-based depression level analysis by encoding deep spatiotemporal features," in IEEE Transactions on Affective Computing, vol. 12, no. 1, pp. 262-268, doi: 10.1109/TAFFC.2018.2870884.

[29] Y. Ding, X. Chen, Q. Fu, and S. Zhong, "A depression recognition method for college students using deep integrated support vector algorithm," IEEE-Access-Special Section On Emerging Deep Learning Theories And Methods For Biomedical Engineering, vol. 8, pp. 75616-75629, 2020.

[30] M. M. Tadesse, H. Lin, B. Xu, and L. Yang, "Detection of depression-related postsin reddit social media forum," IEEE Access, vol. 7, pp. 44883-44893, 2019, doi: 10.1109/ACCESS.2019.2909180

[31] B. Cao, L. Zheng, C. Zhang, P. S. Yu, A. Piscitello, and J. Zulueta, "DeepMood: modeling mobile phone typing dynamics for mood detection," arXiv:1803.08986v1[cs.HC], Mar. 2018.

[32] N. Mahendran, P. M. D. R. Vincent, K. Srinivasan, V. Sharma, and D. N. K. Jayakody, "Realizing a stacking generalization model to improve the prediction accuracy of major depressive disorder in adults," IEEE Access, vol. 8, pp. 49509-49522, 2020, doi: 10.1109/ACCESS.2020.2977887. 


\section{BIOGRAPHIES OF AUTHORS}

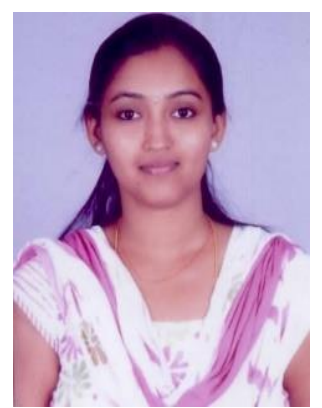

Yashaswini Kunjali Ajeeth Kumar (D) SC P pursuing her Ph.D. in Presidency University. Completed M. Tech (Computer Network and Engineering) in National Institute of Engineering (VTU) in the year 2011 and BE (Information Science and Engineering) in RLJIT (VTU) in the year 2009. Having a 10 years of teaching experience, she has served as Assistant Professor in Maharaja Institute of Technology at Mysore from 2012-2018 and as a Lecturer at SJCIT, Chikaballapur in the year 2011. Currently, working as Assistant Professor in the Department of Computer Science and Engineering, School of Engineering, Presidency University, Bangalore, she is doing her research in Data Analytics and Machine Learning. She has presented a literature paper on Bipolar Disorder: A Pathway Towards Research Progress in Identification and Classification. In: Silhavy R. (eds) Intelligent Algorithms in Software Engineering. CSOC 2020. Advances in Intelligent Systems and Computing, vol 1224. Springer, Cham. She can be contacted at email: yashaswini@ presidencyuniversity.in.

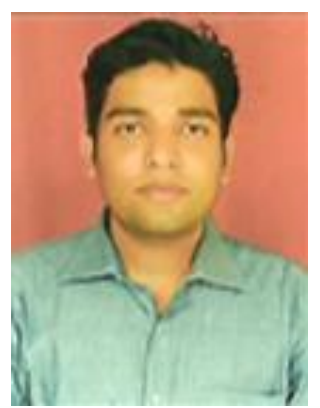

Aditya Kishore Saxena (D) 8d SC P awarded Ph.D. (Computer Science and Engineering) from IIT Allahabad in 2017. His research topic is design and implementation of a framework for age group estimation and his research area is Machine learning and Pattern recognition. Done his MS (Cyber laws and Information Security)-Silver Medalist from IIT Allahabad, UP in 2009. Done his BTech in Computer Science from Uttar Pradesh Technical University, Lucknow in 2007. He can be contacted at email: adityasaxena@ presidencyuniversity.in. 